\title{
ESTUDO DE REFINAMENTO DA MALHA EM REGIÕES DE UM DUTO PARCIALMENTE OBSTRUÍDO E ANÁLISE DA CONVERGÊNCIA DE TENSORES UTILIZANDO SIMULAÇÃO NUMÉRICA
}

\author{
F. RODRIGUES SANTOS ${ }^{1,2}$, G. da SILVA $\operatorname{COSTA}^{1,2}$, A. T. da CUNHA LIMA ${ }^{3,4}$ e I. C. da \\ CUNHA LIMA ${ }^{1,4,5}$ \\ ${ }^{1}$ Centro Universitário SENAI-Cimatec - Salvador/ BA/Brasil, \\ ${ }^{2}$ Instituto Federal da Bahia, Campus de Camaçari - Camaçari/BA/Brasil \\ ${ }^{3}$ Instituto de Física, Universidade Federal da Bahia - Salvador/BA/Brasil \\ ${ }^{4}$ Instituto Nacional de Ciência e Tecnologia-Geofísica do Petróleo, Salvador/BA/Brasil \\ ${ }^{5}$ Pursuelife - Consultancy on Applied Science, Salvador/BA/Brasil \\ E-mail para contato: rfabio10@gmail.com
}

\begin{abstract}
RESUMO - É conhecido da literatura que a dinâmica de um escoamento é afetada pelo surgimento de vórtices atrás de obstáculos sugeridos ao fluxo. Utilizando o OpenFOAM, foram criadas algumas geometrias de dutos em 2-D com variações de obstáculos onde, através do cálculo da média e do desvio padrão realizou-se um estudo de malha estabelecendo refinamentos que indicam convergência no cálculo de alguns tensores com menor esforço computacional. O artigo mostra também a grande necessidade de estabelecer estudos de malha para obtenção de resultados mais precisos e que não apresentem divergência nas soluções numéricas.
\end{abstract}

\section{INTRODUÇÃO}

Os movimentos dos líquidos e gases trazem um conceito que descreve como se comportam os fluidos. A Dinâmica dos Fluidos é o ramo da ciência aplicada que se preocupa em estudar e entender tais movimentos. Alguns movimentos são denominados de turbulentos, caracterizados por apresentarem flutuações de pressão e velocidade. A turbulência é responsável pelo aumento na resistência a um escoamento, pois tudo se passa como se a viscosidade fosse maior, mas ela também reduz o arrasto de um corpo ao retardar o ponto onde ocorre a separação da camada limite (Schlichting, 2000). Osborne Reynolds (1883), através da injeção de corante em um escoamento de água em um duto circular observou os diferentes regimes de escoamento, iniciando com o escoamento laminar, onde as camadas de fluidos deslocam-se umas sobre as outras como se fossem lâminas. À medida que a velocidade do escoamento era aumentada, surgiam instabilidades na forma de oscilações no escoamento até causar a mistura completa do corante com a água, devido à presença de flutuações de velocidade transversalmente à direção principal do escoamento. Este movimento que causa a mistura é chamado de turbulência.

Os estudos aqui realizados em CFD (Computational Fluid Dynamics) utilizaram o OpenFOAM (Greenshields, 2015), que é um software de código aberto para solução numérica 


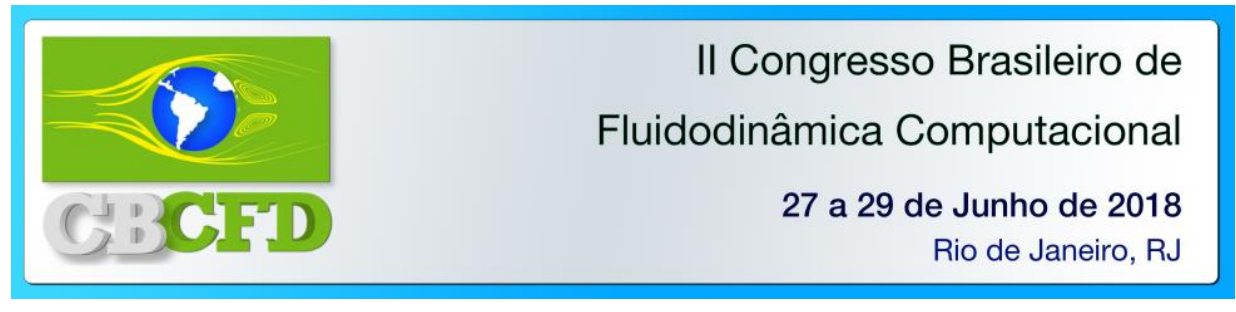

de equações diferenciais, usando o Método dos Volumes Finitos, em particular, obtenção de soluções numéricas das Equações de Navier-Stokes, onde foram simuladas geometrias em dutos 2-D com algumas variações de obstáculos. De modo geral, por Greenshields (2015), o OpenFOAM é um conjunto de bibliotecas $\mathrm{C}++$ projetado para resolver problemas complexos em mecânica dos fluidos, devido à sua flexibilidade que permite o desenvolvimento de agentes de resoluções especificadas pelo utilizador. Dentro de suas bibliotecas, o OpenFOAM integra turbulência nos modelos (RANS e LES), modelos termofísicos, modelos de radiação e as funções de parede, que podem ser acessados ao desenvolver um solver. Sua metodologia de utilização consiste na geração de malhas e manipulação dos parâmetros de entrada, para posterior solução numérica do problema através do modelo físico-matemático e das equações governantes. Depois de realizados os cálculos, parte-se para a visualização da simulação através do programa Paraview.

É neste sentido que o presente artigo se propõe realizar um estudo de malha em regiões próximas às paredes de um duto, através de efeitos causados na natureza da turbulência induzida por obstruções (Patil e Tiwari, 2008), visando identificar um refinamento mínimo de convergência no que diz respeito à confiabilidade da simulação e viabilidade no poder computacional.

\section{GERAÇÃO DA MALHA}

A fase inicial consiste na definição do domínio e geração das malhas do perfil analisado utilizando pré-processador de malhas estruturadas, blockMesh, do OpenFOAM. Foi estabelecido um modelo de domínio com dois obstáculos simétricos de 0,03 $\mathrm{m}$ cada, próximo à entrada. A simulação em 2-D consiste em um duto de seção transversal retangular com 5,1 $\mathrm{m}$ de comprimento $(\mathrm{x})$ sendo $0,09 \mathrm{~m}$ antes da obstrução, $0,01 \mathrm{~m}$ de obstrução e 5,0 m após a obstrução, largura de $0,1 \mathrm{~m}$ (y) e profundidade de $0,01 \mathrm{~m}(\mathrm{z})$. As malhas são divididas em zonas de refinamento, onde a zona de maior refinamento encontra-se próximo às paredes do duto com $1,0 \mathrm{~cm}$ de largura e com subdivisões de $0,5 \mathrm{~cm}$ em (x). Nesta região é realizado um estudo no refinamento desta zona com o intuito de observar maiores variações de velocidades na camada limite, como vemos na figura 1 .

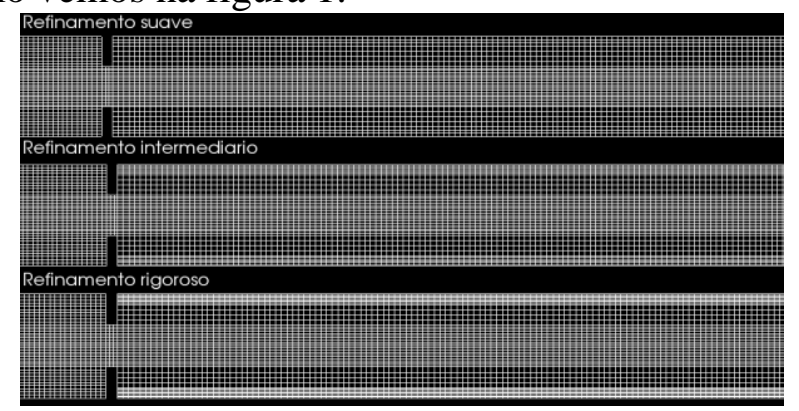

Figura 1 - Duto com obstáculos simétricos com zonas de maior refinamento próximo às paredes, sendo o duto de baixo com as zonas mais refinadas, o do meio a intermediaria e a de cima, a menos refinada

Foram analisados três tipos de refinamentos nesta zona, com 3, 6 e 9 subdivisões. Utilizando a geometria de obstáculos simétricos calculamos a média e o desvio padrão em 30 pontos, sendo 20 em dois eixos dentro da zona de maior refinamento, 10 em cada parede e 10 


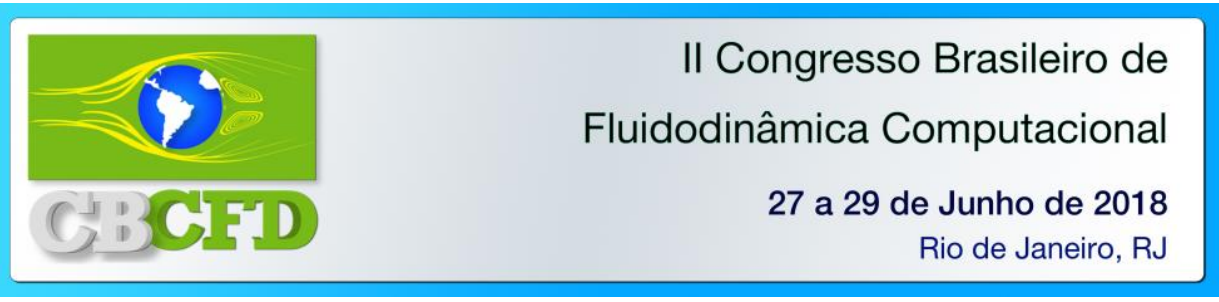

em um eixo central do duto usando um refinamento com subdivisões de $0,5 \mathrm{~cm}$ em (x) e 0,2 $\mathrm{cm}$ em (y), como mostrado na figura 2.
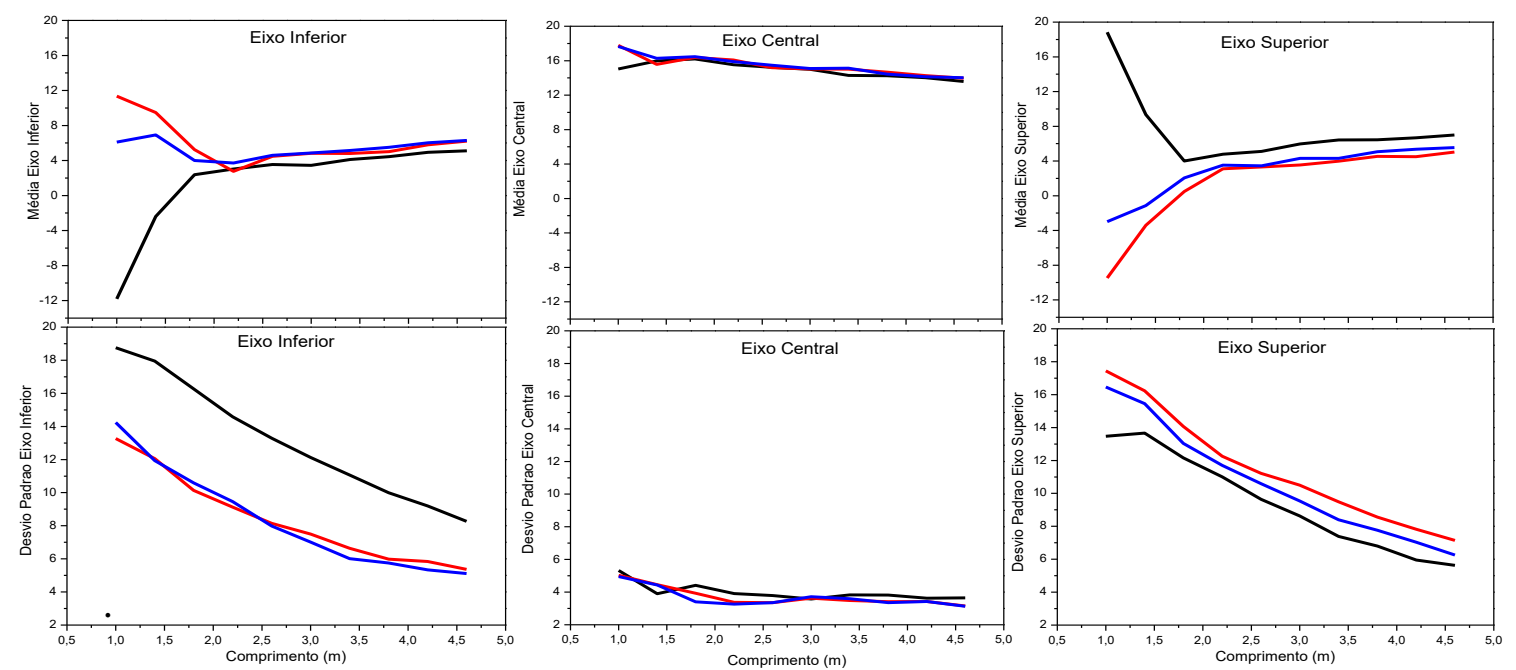

Figura 2 - Calculo da média e desvio padrão para os eixos inferior, central e superior. Cor preta 3 subdivisões, vermelha 6 subdivisões e azul 9 subdivisões nas zonas próximas à parede

A figura 2 mostra um escape da curva preta, até 2,0m, na média nos eixos inferior e superior. Já no desvio padrão é percebido um significativo afastamento na curva preta em relação às demais para o eixo inferior indicando uma distribuição mais achatada. Para está simulação o eixo central não sofreu muita influencia dos refinamentos nas paredes tanto na média como no desvio padrão.
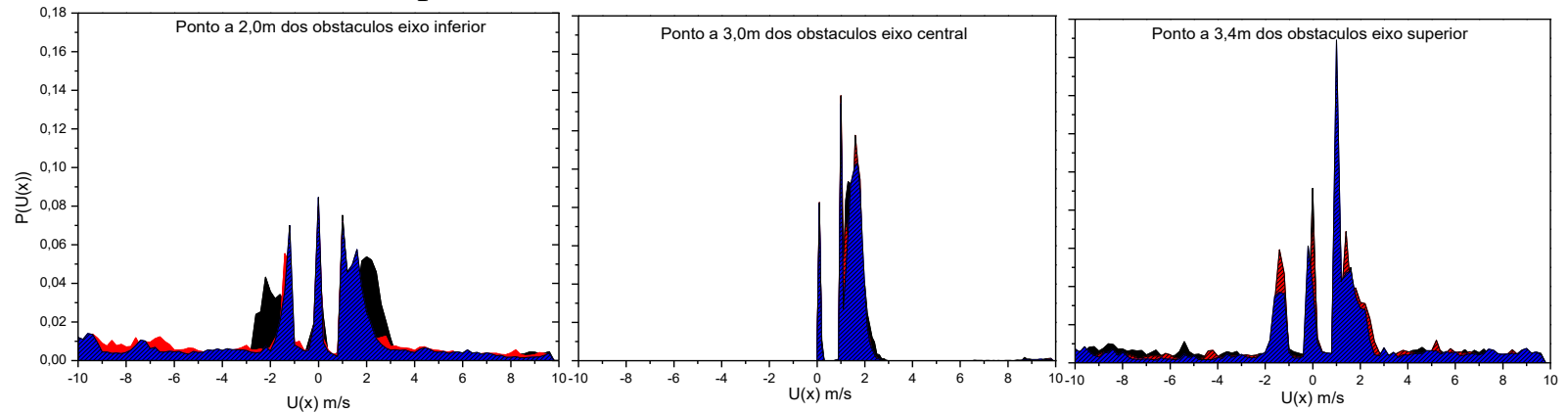

Figura 3 - Distribuição de probabilidade da velocidade Ux em pontos nos 3 eixos. Cor preta 3 subdivisões, vermelha 6 subdivisões e azul 9 subdivisões nas zonas próximas a parede

A figura 3 reforça a análise anterior mostrando que a distribuição na cor preta se diferencia das demais pelo menos em dois trechos no eixo inferior. Os outros dois eixos não apresentam diferenças significativas na distribuição. Seguindo este estudo através da media, desvio padrão e da distribuição, adotou-se 6 subdivisões em y nas zonas próximas as paredes.

\section{ANÁLISE VISUAL DE VARIAÇÕES DE TENSORES A PARTIR DO REFINAMENTO DA MALHA}

Em busca de uma análise visual da simulação após o estudo estatístico da seção 2, partiu-se para verificar o comportamento do escoamento através da velocidade e da vorticidade. A figura 4(a) mostra o vetor velocidade e a figura 4(b) a vorticidade (z) nos três 


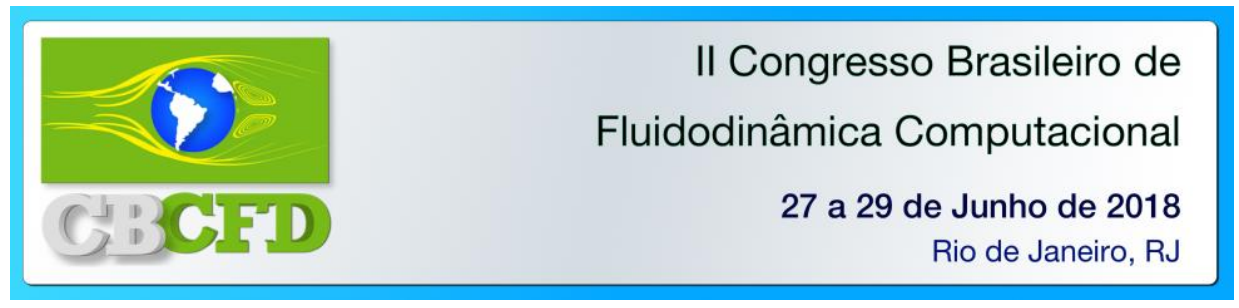

modelos de refinamentos citados na seção 2 , refinamento suave (3 subdivisões), refinamento intermediário (6 subdivisões) e refinamento rigoroso ( 9 subdivisões).

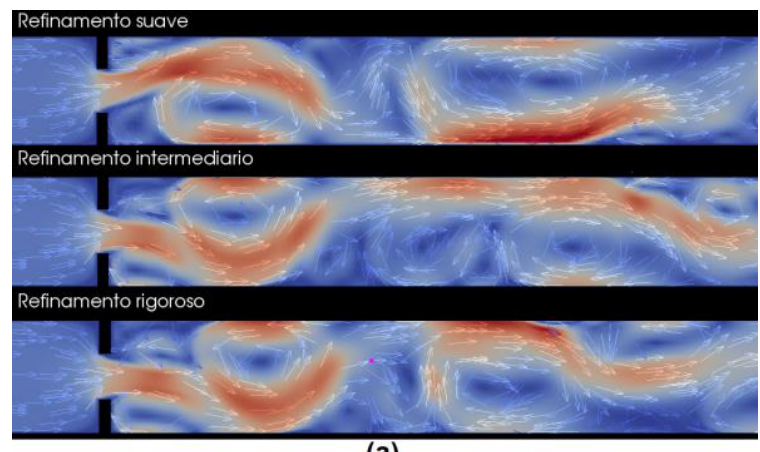

(a)

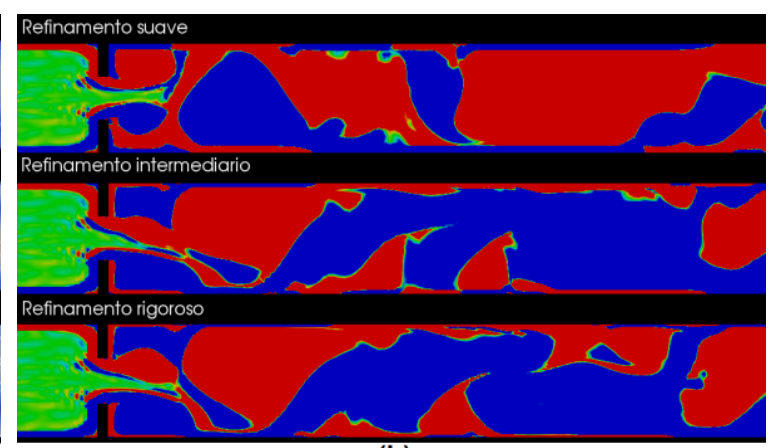

(b)

Figura 4 - Instantâneo da simulação de um fluxo a $5 \mathrm{~m} / \mathrm{s}$ em três refinamentos próximos a parede, onde (a) representa o modulo da velocidade e (b) a vorticidade

Nota-se um fluxo diferenciado na figura 4(a) para o refinamento suave em relação aos outros dois em quase todo trecho do instantâneo, onde é possível visualizar vetores em sentido contrário. De mesma forma a figura 4(b) os refinamentos intermediários e rigorosos se assemelham em relação ao refinamento suave, a cor vermelha indica vorticidade positiva e praticamente se igualam para os refinamentos intermediários e rigorosos o mesmo ocorre para a cor azul que indica vorticidade negativa. Do modo praticamente contrário, as cores no refinamento suave se diferenciam dos outros dois, sugerindo, ainda que aqui ilustrado por um instante de tempo, uma distinção no cálculo dos tensores pela malha menos refinada e uma aproximação (convergência) de valores quando o refinamento é intensificado.

\section{CONCLUSÃO}

Foram realizados estudos de malha próximo às paredes identificando refinamentos que conduzam à convergência e validem a simulação com menor esforço computacional. Para este modelo estudado de um fluxo incompressível a $5 \mathrm{~m} / \mathrm{s}$ através dos instantâneos do vetor velocidade e da vorticidade, percebeu-se que a malha menos refinada destoa das outras duas que mantêm um comportamento bem semelhante em quase todo duto. Reforçado pelos cálculos estatísticos da média e desvio padrão percebeu-se que os refinamentos em 6 e 9 subdivisões se assemelham em quase um colapso das curvas. Sendo assim, o estudo estabelece como o refinamento 6 , para zonas próximas às paredes, como suficiente para captar as variações de tensores como velocidade e vorticidade para tal geometria.

\section{REFERÊNCIAS}

GREENSHIELDS, C. J. OpenFOAM. The Open Source CFD Toolbox User Guide, 2015.

PATIL, PRATISH P.; TIWARI, SHALIGRAM. Effect of blockage ratio on wake transition for flow past square cylinder. Fluid Dynamics Research, v. 40, n. 11, p. 753-778, 2008.

REYNOLDS, O. 1883. An experimental investigation of the circunstances which determine whether the motion of wather shall be direct of sinous, and of the law of resistence in parallel channels. Philos. Trans. R. Soc., London 174, pp. 935-982.

SCHLICHTING, H.; GERSTEN, K., Boundary Layer Theory, 2000 8th edition Springer. 